\title{
Fundamental Aspects of Magnetic Shape Memory Alloys: Insights from $a b$ initio and Monte Carlo Studies
}

\author{
Peter Entel ${ }^{1, \mathrm{a}}$, Markus E. Gruner ${ }^{1}$, Antje Dannenberg ${ }^{1}$, Mario Siewert ${ }^{1}$, \\ Sanjeev K. Nayak ${ }^{1}$, Heike C. Herper ${ }^{1}$, and Vasiliy D. Buchelnikov ${ }^{2}$ \\ ${ }^{1}$ Physics Department, University of Duisburg-Essen, 47048 Duisburg, Germany \\ ${ }^{2}$ Physics Department, Chelyabinsk State University, Chelyabinsk 454021, Russia \\ aentel@thp.uni-duisburg.de
}

Keywords: Ferromagnetic Heusler alloys. Magnetic shape memory effect. Ab initio calculations.

\begin{abstract}
Ferromagnetic Heusler alloys like Ni-Mn-Z (Z = Al, Ga, In, Sn, Sb), which undergo a martensitic phase transformation, are on the edge of being used in technological applications involving actuator and magnetocaloric devices. The other class of ferromagnetic full Heusler alloys like Co-Mn-Z (Z = Al, Si, Ga, Ge, Sn) not undergoing a structural phase transition, are half-metals (in contrast to the Ni-based systems) with high spin polarization at the Fermi level and are of potential importance for future spintronics devices. On the basis of recent $a b$ initio calculations, we highlight the main differences between the two classes of Heusler based materials.
\end{abstract}

\section{Introduction}

Since the pioneering work of Webster et al. [1, 2], there has been new interest in a particular class of ferromagnetic (FM) shape memory alloys (MSMA), which undergo a structural transformation from the FM high-temperature cubic $\mathrm{L} 2$ phase to the tetragonally distorted $5 \mathrm{M}$ or $7 \mathrm{M}$ phase, which shows an extremely large strain of up to $10 \%$ in an external field of less than 1 Tesla [3-6]. This is the basis of the one- and two-way shape memory effect (SME) due to magnetically controlled martensitic transformation as experimentally observed [7]. By now, these magneto-induced shape memory mechanisms have in detail been investigated for mono- and polycrystalline Ni-Mn-Fe-Ga samples [7-9] including twin boundary motion in the modulated phases under the impact of the external magnetic field [10].

Nevertheless, fundamental questions regarding the energetics of twin boundary motion in relation to the local atomic structure, chemical composition, magnetic state and magnetic anisotropy as well as the electronic structure (see Ref. [11] for an overview) have still to be worked out. What remains to be done from a practical point of view regarding the use of MSMA and magneto-caloric (MCE) devices is to find new FM Heusler alloys which allow higher working temperatures.

Regarding Heusler-based spintronics materials like Co-Mn-Fe-Si, the practical point of view in relation to application purposes is to maintain half-metallicity in the samples to achieve high magnetoresistance values at room temperatures. From a theoretical point of view the realization of half-metallic Heusler alloys, with a $100 \%$ spin polarization at the Fermi level depends on details of the electronic structure and the valence electron number per atom, $e / a$, limiting the possible candidates, for instance, see Galanakis et al. [12, 13]. Information on the properties of spintronics materials may be taken from Refs. [14, 15].

In this paper, we present a few new basic results of both types of Heusler alloys, based on $a b$ initio calculations and finite temperature simulations using statistical models like the Heisenberg and $q$-state Potts model [16]. Although the emphasis in this paper mainly concerns MSMA, a simultaneous discussion of electronic and magnetic features of spintronics materials gives a deeper understanding of basic features determining the behavior of both types of Heusler systems, i.e., martensitically unstable (MSMA) and stable FM Heusler alloys (suited for spintronics applications). We will show that the number of valence electrons per atom, $e / a$, in conjunction with the variation of the spin magnetic moments with change in temperature are decisive parameters when considering the martensitic instability in MSMA. 


\section{First-Principles Calculations of the Electronic and Magnetic Properties of Heusler Alloys}

There is a series of electronic structure calculations of MSMA and spintronics materials available from the literature, for example, see Refs. [11, 12, 17-25], emphasizing the importance of a band Jahn-Teller effect for the structural instability (martensitic transformation) in MSMA and the existence of a band gap around the Fermi energy, $E_{F}$, in the minority-spin channel of materials of interest for spintronics.

We have repeated some of the electronic structure calculations using VASP [26, 27] and show in Fig. 1 and 2 the resulting spin-down band structures of the spintronics material $\mathrm{Co}_{2} \mathrm{MnGe}$ and the MSMA Ni ${ }_{2} \mathrm{MnGa}$ with element-specific projections (left) and symmetry assigned bands (right).
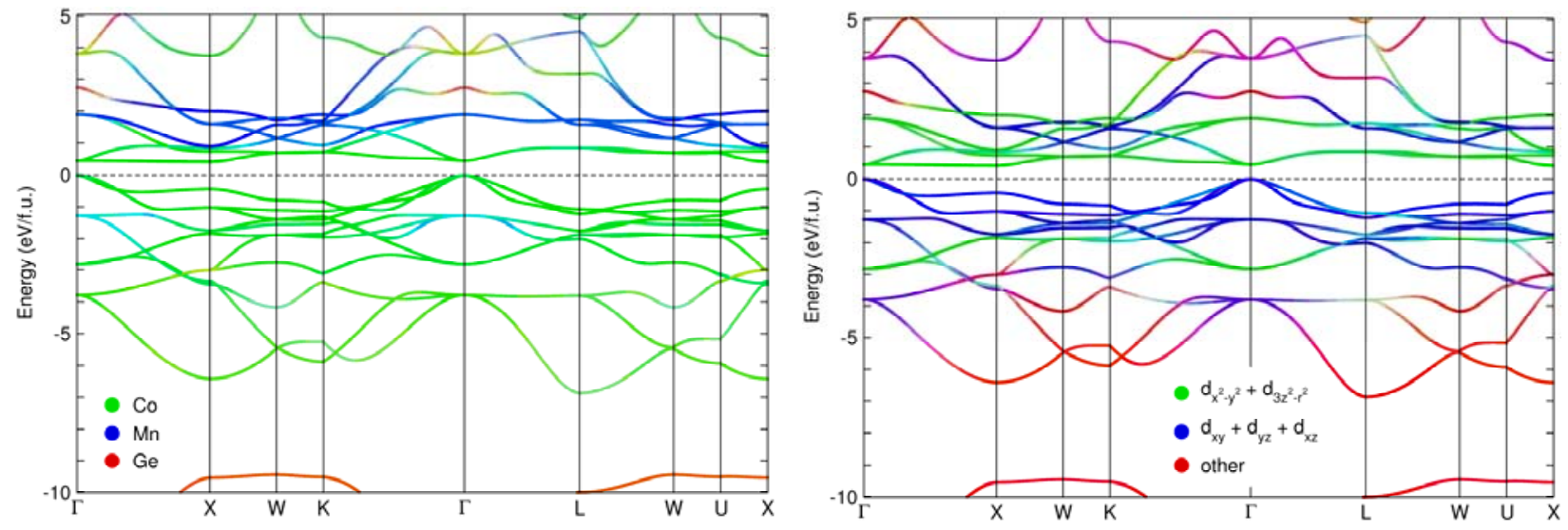

Fig. 1: Minority-spin band structure of $\mathrm{Co}_{2} \mathrm{MnGe}$ projected onto the element-specific contribu-tions (left) and symmetry-assigned contributions (right), showing the formation of the half-metallic gap around the Fermi energy.
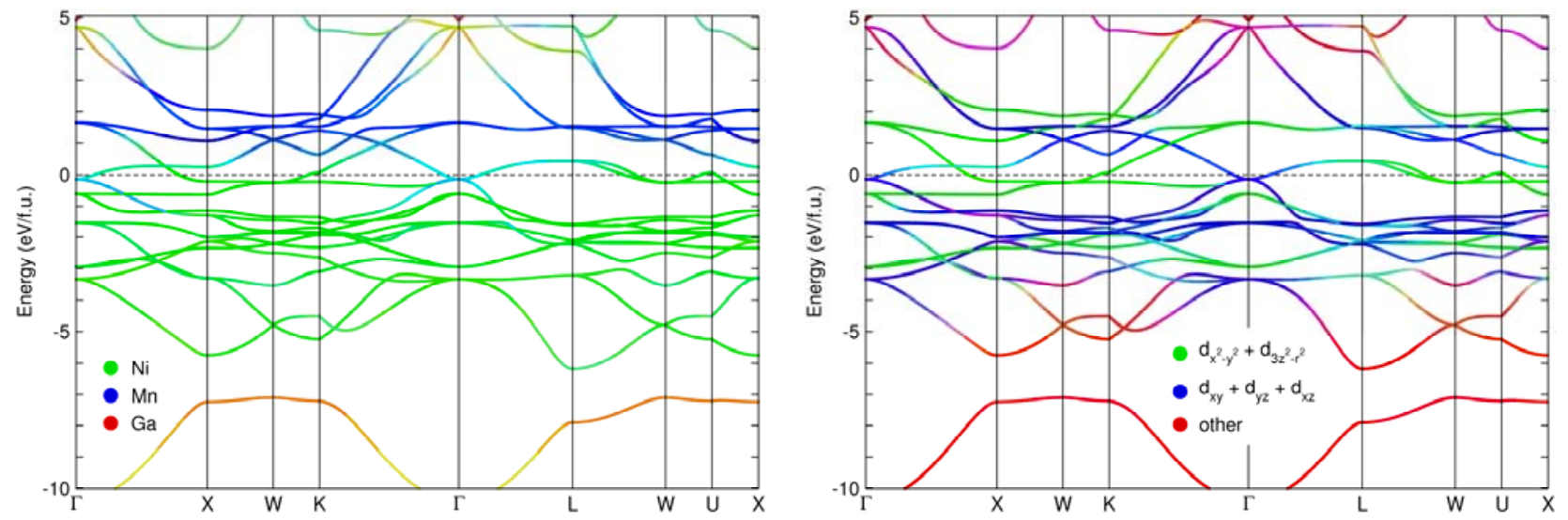

Fig. 2: Minority-spin band structure of $\mathrm{Ni}_{2} \mathrm{MnGa}$ projected onto the element-specific contributions (left) and symmetry-assigned contributions (right), showing difference that arises compared to the case of $\mathrm{Co}_{2} \mathrm{MnGe}$ like the closing of the energy gap at $E_{F}$.

We find in the majority-spin bands either Co or Ni and Mn near the Fermi energy $E_{F}$, while in the minority-spin bands, the states in the vicinity of $E_{F}$ are - apart from the $\Gamma$ point - nearly entirely Co- or Ni- $d$ states of $e_{g}$ symmetry. This scenario leads in case of $\mathrm{Ni}_{2} \mathrm{MnGa}$ to the martensitic instability.

In Fig. 3, the electronic density of states (DOS) curves are depicted showing the formation of a gap in the minority-spin channel of $\mathrm{Co}_{2} \mathrm{MnGe}$ of the order of $0.4 \mathrm{eV}$ yielding a half-metal at zero temperature, while for $\mathrm{Ni}_{2} \mathrm{MnGa}$, this gap is partially filled with $\mathrm{Mn}$ - and $\mathrm{Ni}-e_{g}$ and $t_{2 g}$ states and is pushed below $E_{F}$ by $0.75 \mathrm{eV}$, because of the filling of the band structure by one additional valence 
electron per formula unit as compared to $\mathrm{Co}_{2} \mathrm{MnGe}$.

Note that in $\mathrm{Ni}_{2} \mathrm{MnGa}$, we find a pronounced peak of Ni-eg states in the minority-spin DOS right below $E_{F}$, see Fig. 3. This is a highly unstable situation and the system can lower its energy by a slight tetragonal distortion which pushes this Ni- $e_{g}$ peak by approximately $1 \mathrm{eV}$ above $E_{F}$ and redistributes the electrons in lower energy states. This effectively pushes the gap-like structure towards $E_{F}$ (but does not yield a half-metallic state because there are states in the gap filled by mainly Ni-d electrons). This Jahn Teller like effect (which is first discussed by Ayuela et al. by means of first-principles DOS calculations [19]) is in the present case associated with $e_{g}$ states and not with the $t_{2 g}$ states. In other systems this often leads to a triplet ground state of ${ }^{3} \mathrm{t}_{1 \mathrm{u}}$ configuration which is known to be unstable against a Jahn-Teller distortion [28, 29] (note also that the actual symmetry assignment of the lower and upper gap states in $\mathrm{Co}_{2} \mathrm{MnGe}$ is $t_{1 u}$ (occupied) and $e_{u}$ (unoccupied) [13].) This electronic scenario is the origin of the martensitic instability in Ni2MnGa occurring at $220 \mathrm{~K}$ in the FM state below the Curie temperature at $376 \mathrm{~K}$.
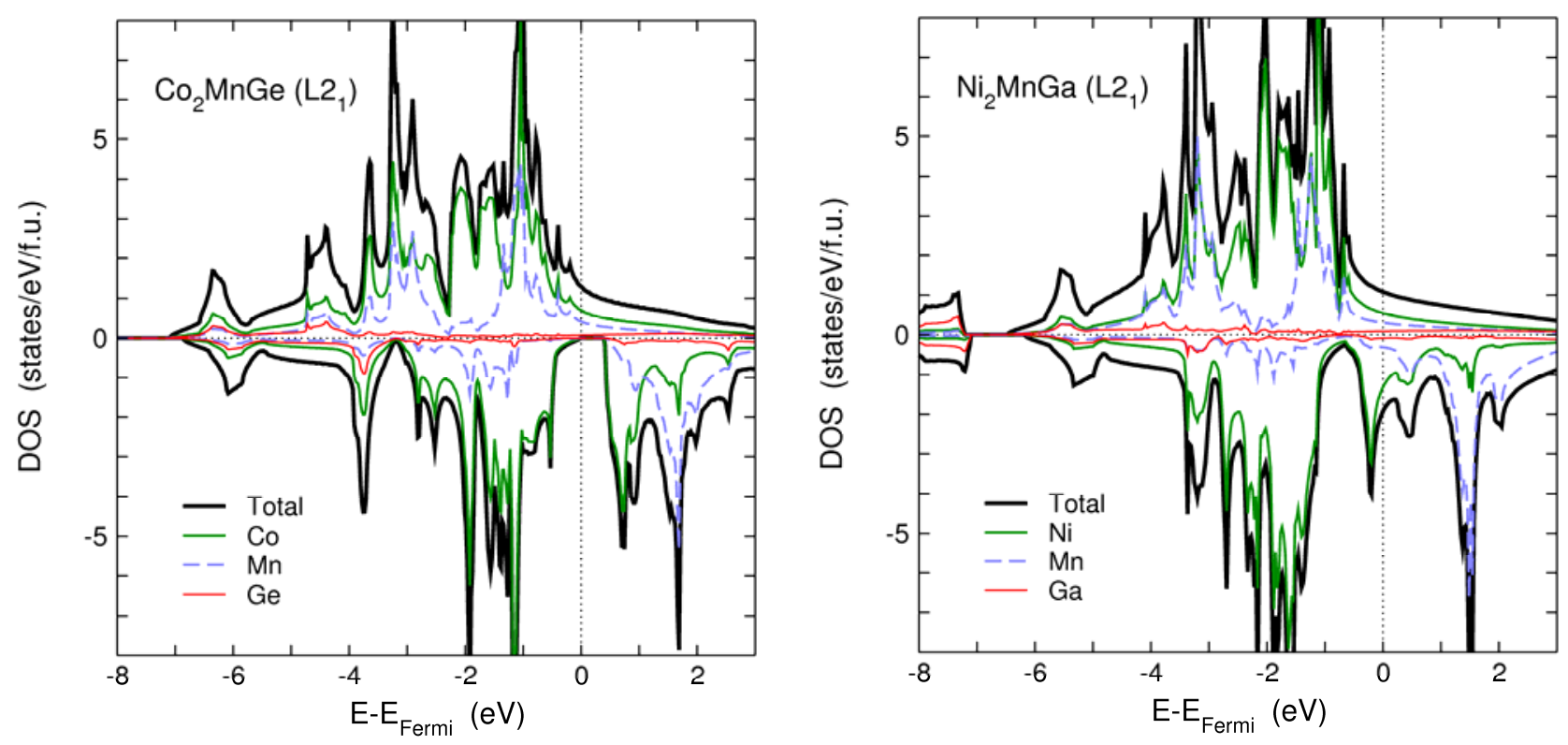

Fig. 3: Total and element-projected electronic density of states curves of $\mathrm{Co}_{2} \mathrm{MnGe}$ (left) and $\mathrm{Ni}_{2} \mathrm{MnGa}$ (right) showing the formation of the energy gap at $E_{F}$ for the Co-based Heusler compound. For the Ni-based system a peak at $E_{F}$ appears which mainly consists of Ni- $e_{g}$ states responsible for the Jahn-Teller like distortion.

Now, the gap of the full-Heusler alloys is still a rather subtle point [19]. When considering $\mathrm{Ni}_{2} \mathrm{MnGa}$ in distinction to $\mathrm{Co}_{2} \mathrm{MnGe}$, a close look at the behavior of the minority-spin band structure reveals that it is not a mere band filling effect but also shows that hybridization of states near $E_{F}$ is significantly different. In both compounds, the Co(Ni) atoms occupy sites of octahedral symmetry which splits the $d$-states into $e_{g}, \mathrm{e}_{u}, t_{2 g}$ and $t_{1 u}$, which are doubly and triply degenerated. In the Co-based Heusler alloy, the fact that the $\mathrm{e}_{u}$ and $t_{1 u}$ states arising from octahedral symmetry cannot hybridize with the Mn- $d$ or Ge-p states (with Mn and Ge sitting at the tetrahedral sites of the $\mathrm{L} 2{ }_{1}$ crystal structure) together with the right valence electron number of $e / a=7.25$ (in contrast to 7.5 in case of $\mathrm{Ni}_{2} \mathrm{MnGa}$ ) causes the formation of an energy gap, whereby $E_{F}$ comes to lie between the $\mathrm{e}_{u}$ and $t_{1 u}$ levels [19]. Due to this half-metallicity, the spin polarization $P$ at $E_{F}$ arises solely from the majority-spin states, i.e., $P=100 \%$, while $P=-25.6 \%$ in case of $\mathrm{Ni}_{2} \mathrm{MnGa}$. It would be interesting to know whether FM Heusler alloys were possible, which exhibit the MSME as well as a 
large $P$ value (although in general, large $P$ may mean low Fermi surface nesting inhibiting modulated phases). If we consider the critical values $e / a=7.25\left(\mathrm{Co}_{2} \mathrm{MnGe}\right)$ and $e / a=7.5$ $\left(\mathrm{Ni}_{2} \mathrm{MnGa}\right)$ one may conceive an alloy of composition CoNiMnGe${ }_{0.5} \mathrm{Ga}_{0.5}$ with $e / a=7.375$ in between a half-metallic and metallic system. Recent $a b$ initio calculations of a similar system, CoNiMnGa with $\mathrm{L}_{1}$-like structure and $e / a=7.25$, shows the existence of a gap right below $E_{F}$ (and a larger spin polarization than $\mathrm{Ni}_{2} \mathrm{MnGa}$ ) and no tendency for a martensitic instability. The corresponding DOS is shown in Fig. 4. In a very similar way, one may systematically try to obtain new Heusler alloys of interest for spintronics as well as MSM devices by combining systems from the series Co-Mn-Z ( $\mathrm{Z}=\mathrm{Al}, \mathrm{Si}, \mathrm{Ga}, \mathrm{Ge}, \mathrm{Sn})$ with those from Ni-Mn-Z ( $\mathrm{Z}=\mathrm{Al}$, Ga, In, Sn, Sb).
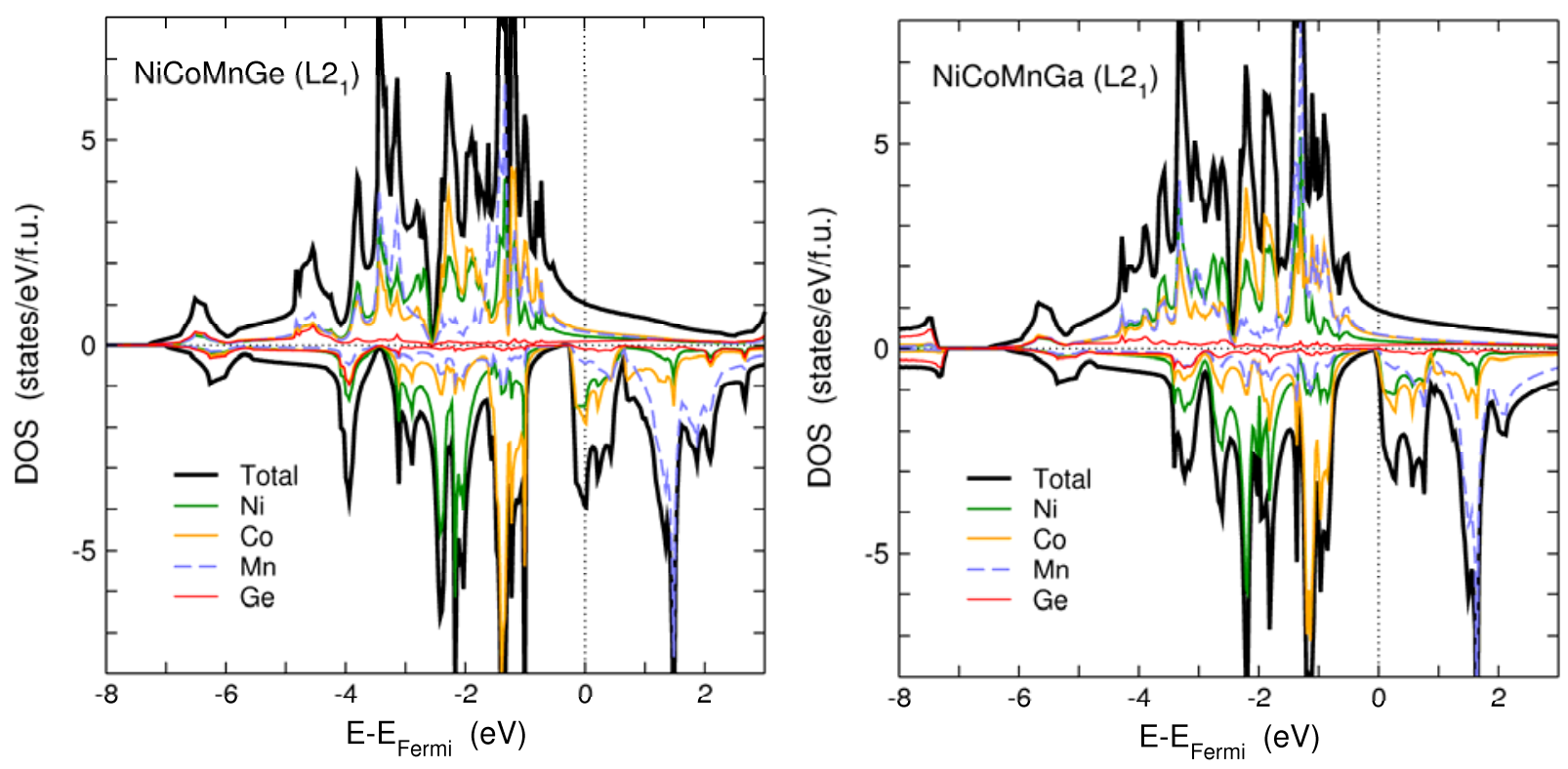

Fig. 4: Density of states of cubic NiCoMnGe $(e / a=7.5)$ and NiCoMnGa $(e / a=7.25)$ - close to half-metallic behavior; the latter system does not show tendency for a martensitic instability.

\section{Origin of Martensitic Transformation in Ni-Mn-Z (Z = Al, Ga, In, Sn, Sb) Heusler Alloys}

The ferromagnetic Heusler systems Ni-Mn-Z ( $\mathrm{Z}=\mathrm{Al}, \mathrm{Ga}, \mathrm{In}, \mathrm{Sn}, \mathrm{Sb})$ all exhibit a martensitic instability of which Ni-Mn-Ga is the only one showing the instability at the stoichiometric composition $\mathrm{Ni}_{2} \mathrm{MnGa}$. In fact, near-stoichiometric compounds of $\mathrm{Ni}_{2} \mathrm{MnGa}$ show a series of reversible phase transformations with decreasing temperature from the high-temperature cubic $\mathrm{L} 2_{1}$ structure to premartensitic modulated $3 \mathrm{M}$ and then to the modulated martensitic phases $5 \mathrm{M}$ and $7 \mathrm{M}$ (with a tetragonal distortion $c / a=0.94$ ) and finally to the non-modulated tetragonal $\mathrm{L} 1_{0}$ structure with $c / a=1.26$ at very low temperatures. Such sequence of transitions was reported by Chernenko et al. for three alloys with slightly different off-stoichiometric compositions; for these compositions, the modulated premartensitic phase was not observed. Figure 5 shows the phase diagram of Ni-Mn-Ga for Ni-excess concentration [11] and the schematic phase diagram of Ni-Mn$\mathrm{Z}(\mathrm{Z}=\mathrm{Ga}, \mathrm{In}, \mathrm{Sn}, \mathrm{Sb})$ of the martensitic transformation temperatures as a function of $e / a$ [31].

In case of stoichiometric composition, we find $e / a=7.5\left(\mathrm{Ni}_{2} \mathrm{MnGa}\right.$ and $\left.\mathrm{Ni}_{2} \mathrm{MnIn}\right), e / a=7.75$ $\left(\mathrm{Ni}_{2} \mathrm{MnSn}\right)$ and $e / a=8\left(\mathrm{Ni}_{2} \mathrm{MnSb}\right)$, showing that, apart from $\mathrm{Ni}_{2} \mathrm{MnGa}$, the other MSMA only undergo a structural transformation for off-stoichiometric compositions.

With respect to the origin of the structural instability in the MSMA, attention has to be paid to the possible electronic and phononic driving forces. For simplicity, limiting the discussion to prototype $\mathrm{Ni}_{2} \mathrm{MnGa}$, we observe electrons with $e_{g}\left(e_{u}\right)$ symmetry contributing to the instability by Fermi surface nesting associated with the corresponding peak in the minority DOS, see Fig. 3 and the discussion in Ref. [11]. The system can lower its energy by a redistribution of electrons and 
simultaneous shift of this peak to a higher region above $E_{F}$. The resulting DOS for the stable martensitic 5M $(c / a=0.94)$ and non-modulated $\mathrm{L} 1_{0}(c / a=1.25)$ structures are shown in Fig. 6 .
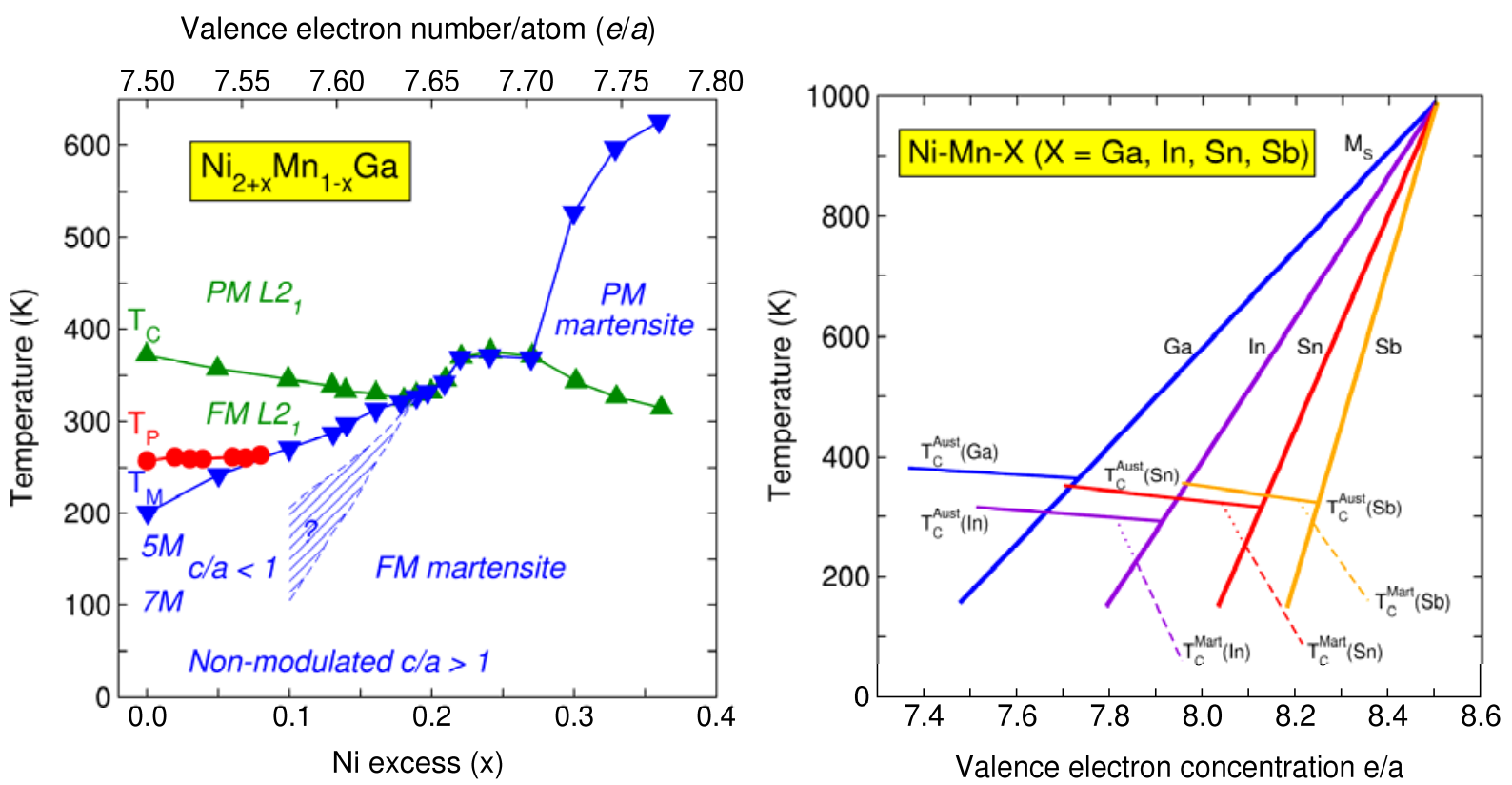

Fig. 5: Phase diagram of Ni-Mn-Ga as a function of Ni-excess concentration (left) showing the Curie temperature $\left(T_{C}\right)$, the premartensitic $\left(T_{P}\right)$ and the martensitic transformation temperature $\left(T_{M}\right)$, respectively [11]. Alloys near stoichiometry show the MSME, whereas alloys with merging $T_{C}$ and $T_{M}$ exhibit the magnetocaloric effect. The schematic behavior of $T_{M}$ as a function of $e / a$ for the Ni-Mn-Z ( $\mathrm{Z}=\mathrm{Ga}, \mathrm{In}, \mathrm{Sn}, \mathrm{Sb})$ alloys is shown in the right panel [31]. The linear behavior of $T_{M}$ with e/a may partially be explained using a rigid band picture (filling in electrons) in conjunction with fixed spin moment calculations, which allow to model the decrease of magnetization with increasing temperature.

The absence of martensitic instabilities in the other alloys of type Ni-Mn-Z (In, Sn, Sb) at stoichiometric composition may tentatively be related to the fact that in this case, we do not find any trace for Fermi surface nesting. Weak nesting behavior appears, for example in case of Ni-Mn$\mathrm{Sb}$, at larger values of $e / a(>8)$ in agreement with the experimental change of $T_{M}$ with $e / a$ in Fig. 5 . However, note that the situation is more complex for the off-stoichiometric alloys, since the shift of $T_{M}$ away from the stoichiometric composition to larger values of $e / a$ is not a mere band-filling effect since hybridization changes in a subtle way around the Fermi level. Also, the influence of phonons on the martensitic transformation may be more important than in case of stoichiometric samples since the lattice becomes elastically softer for the disordered alloys. This is obvious from the behavior of the total energy as a function of tetragonal distortion, $c / a$, showing a change of the energies of cubic and martensitic phases relatively to each other.

However, the situation is more complex, since at $T_{M}=220 \mathrm{~K}\left(\mathrm{Ni}_{2} \mathrm{MnGa}\right)$ the magnetization has decreased and is different from its ground-state value. This pushes the $e_{g}$-peak in the spin-down DOS further away from the Fermi level to lower energies. Since the nesting vectors vary continuously with the magnetization, this means that part of the nesting behavior of the spin-down electrons at $E_{F}$ is lost. However, for this reduced magnetization at finite temperatures we find simultaneously the appearance of a weaker Fermi surface nesting of the majority-spin electrons, which was already discussed in Ref. [32]. So, it seems that Fermi surface nesting in both spin channels is required to provoke the structural transformation from the FM cubic L2 1 phase to the 
modulated premartensitic phase. Since different nesting vectors come from the different spin channels, the premartensitic phase of $\mathrm{Ni}_{2} \mathrm{MnGa}$ has no well defined modulated structure and a reshuffling of the atoms lowers the energy further driving the martensitic transformation to the $5 \mathrm{M}$ structure.
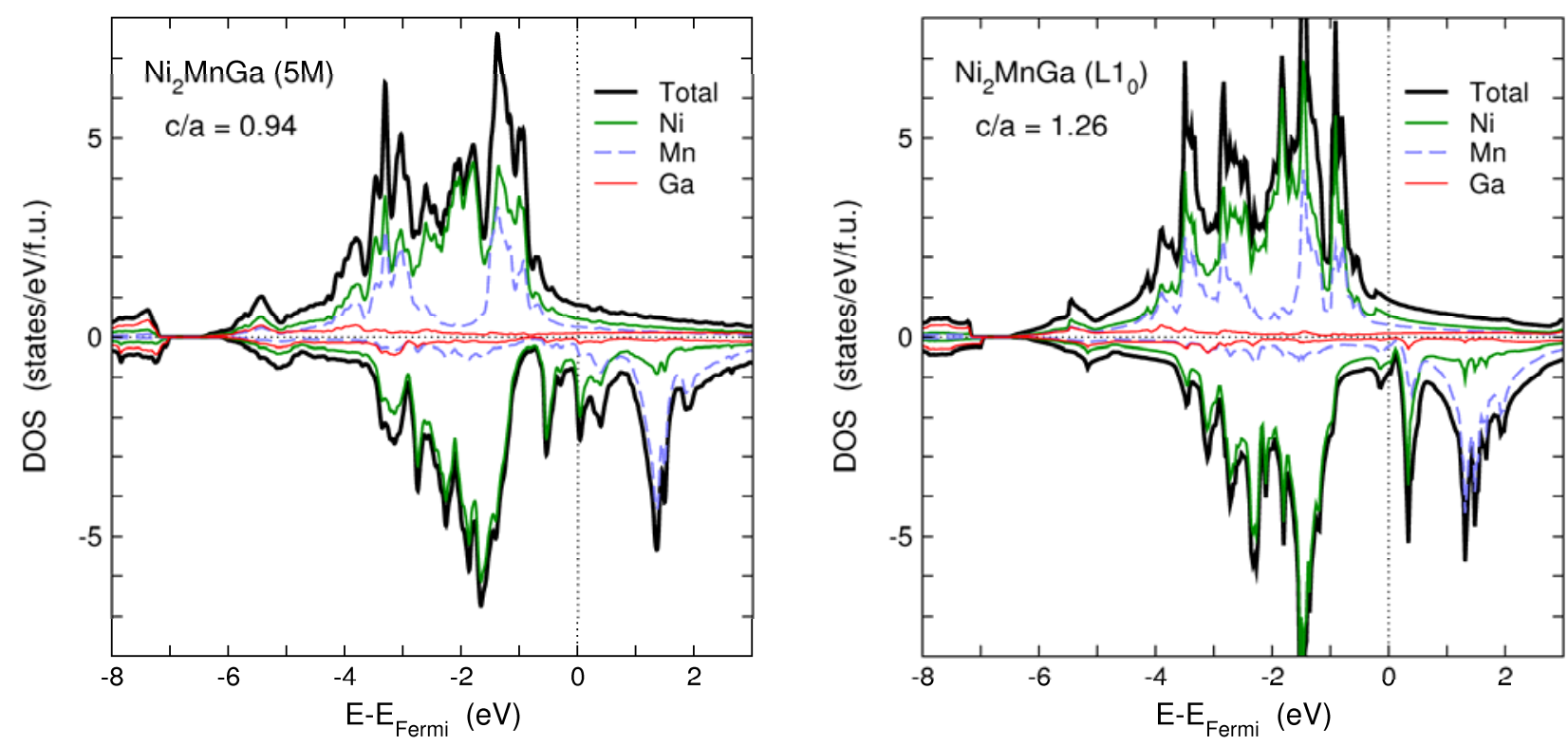

Fig. 6: Total and element-projected electronic density of states curves of $5 \mathrm{M}$ modulated tetragonal $(c / a=0.94) \mathrm{Ni}_{2} \mathrm{MnGa}$ (left) and nonmodulated tetragonal ( $\left.c / a=1.25\right) \mathrm{Ni}_{2} \mathrm{MnGa}$ (right) showing that in both cases the Fermi energy comes to lie at a relatively favorable position.

With respect to the contributions of lattice vibrations to the premartensitic and martensitic transformation in $\mathrm{Ni}_{2} \mathrm{MnGa}$, the situation is even less clear. Phonons start to soften already in the paramagnetic phase and then harden a bit when approaching the premartensitic phase [33-35] and show very unusual behavior below the martensitic phase transition in the 5M phase [36]. The softening was well reproduced in $a b$ initio phonon calculations [37-39]; in particular the firstprinciples calculations of the different contributions to the free energy of $\mathrm{Ni}_{2} \mathrm{MnGa}$ showed that without the magnetic contributions, one fails to get the right sequence of phases at elevated temperatures [40]. Although the displacement fields of the [110] phonons in the cubic L2 1 structure will finally be needed to achieve the transformation to the martensitic phase (with a critical wave vector of $q=0.43$ in [110] direction in case of $\mathrm{Ni}_{2} \mathrm{MnGa}$ ), we believe that the intrinsic instability is related to the simultaneous appearance of nesting behavior in both spin channels in a narrow temperature range fixed by the magnetization of the sysem.

\section{Competing Magnetic Ordering in Ni-Mn-Z (Z = Al, Ga, In, Sn, Sb) Heusler Alloys}

Evaluation of magnetic exchange coupling constants of the ferromagnetic Heusler alloys Ni-Mn-Z ( $\mathrm{Z}=\mathrm{Al}, \mathrm{Ga}, \mathrm{In}, \mathrm{Sn}, \mathrm{Sb}$ ) hints towards a rather complex scenario of competing antiferromagnetic (AF) and FM interactions in the alloys in contrast to half-metals as $\mathrm{Co}_{2} \mathrm{MnGe}$ for which the interactions are dominantly ferromagnetic. Intriguingly, this competition of different magnetic ordering tendencies leads to the appearance of multiple magnetic phases as is the case for Ni-Mn-Sb $[16,41]$. Typically, the magnetization as a function of temperature, $M(T)$, for the off-stoichiometric alloys Ni-Mn-Z ( $\mathrm{Z}=\mathrm{In}, \mathrm{Sn}, \mathrm{Sb})$ shows with decreasing temperature a sudden increase (as in a firstorder like transition) from paramagnetic (PM) austenite to FM austenite and a sudden decrease accompanying the martensitic transformation at still lower $T$. First-principles calculations of the exchange constants, $J_{i j}$, as a function of the distance between the atoms show that the interaction 
between nearest neighbors Co-Mn or Ni-Mn is strongest in all compounds and alloys and plays a decisive role for the value of $T_{C}$.

However, the situation is more subtle, since any degree of disorder and tetragonal distortion leads to oscillating behavior of $J_{i j}$ with AF and FM interaction, which mainly is responsible for the rather unusual magnetization curves of the MSMA. There is a series of publications dealing with the $a b$ initio evaluation of the $J_{i j}$ using direct and frozen magnon methods, all confirming the oscillating $J_{i j}[16,42-44]$. The general trend of how tetragonal distortions or disorder influence the $J_{i j}$ in contrast to the strictly FM interactions in the stoichiometric $\mathrm{Co}_{2} \mathrm{MnGe}$ and $\mathrm{Ni}_{2} \mathrm{MnGa}$ in the $\mathrm{L} 2_{1}$ structure, is shown in Fig. 7. Now, the unusual trends in $M(T)$ (steep increase and subsequent steep decrease gives rise to the usual and inverse magnetocaloric effect (MCE) [45], which is of technological importance.
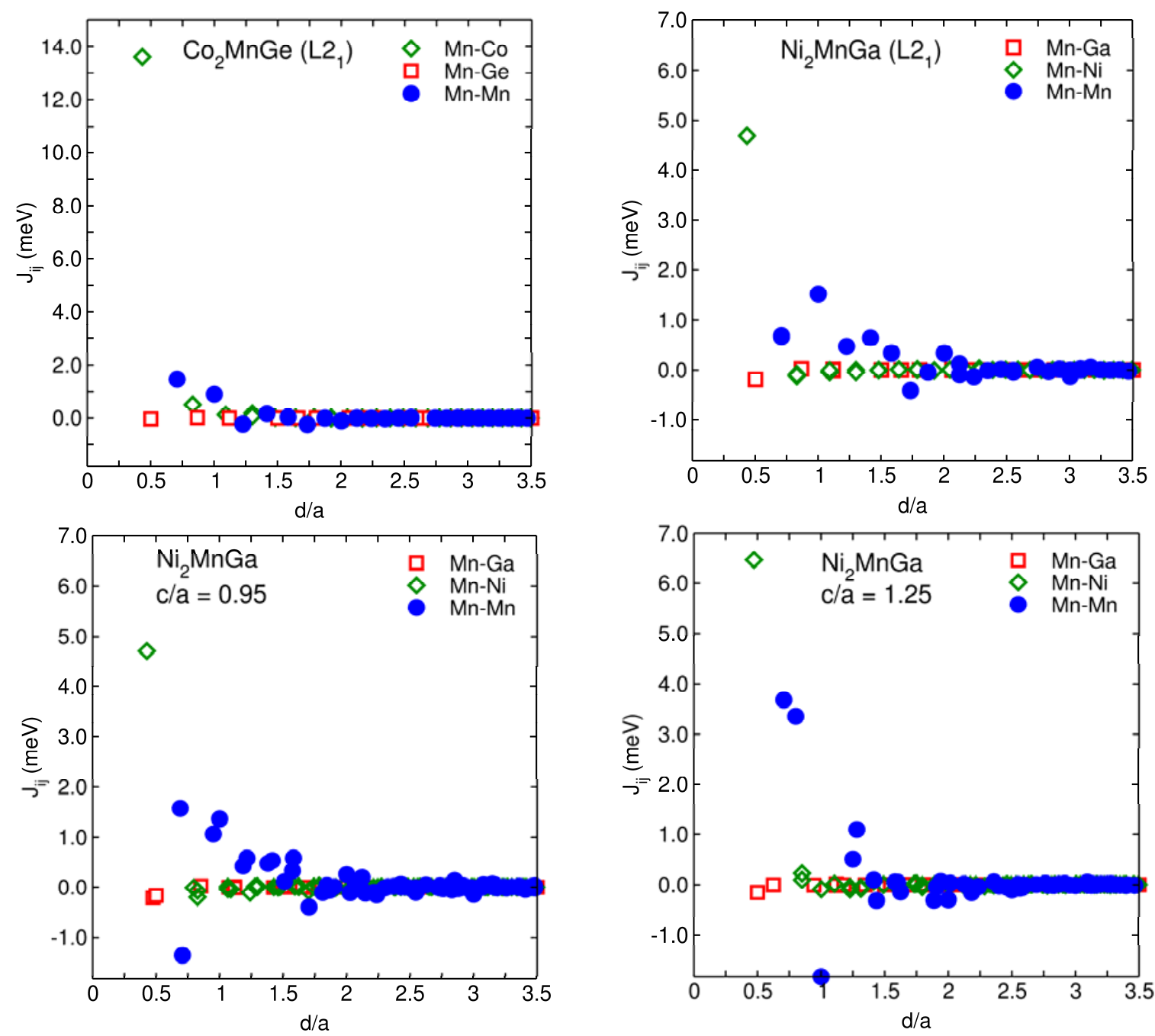

Fig. 7: Magnetic exchange interaction coupling constants $J_{i j}$ as a function of the distance between the atoms in units of the lattice constant for $\mathrm{Co}_{2} \mathrm{MnGe}$ (top left), $\mathrm{Ni}_{2} \mathrm{MnGa}$ (top right), and tetragonally distorted ( $c / a=0.95$ and $c / a=1.25) \mathrm{Ni}_{2} \mathrm{MnGa}$ (lower two figures).

With respect to finite temperature behavior of spintronics materials, the very high Curie temperatures of $\mathrm{Co}_{2} \mathrm{MnGe}\left(T_{C}=900 \mathrm{~K}\right), \mathrm{Co}_{2} \mathrm{MnSi}\left(T_{C}=985 \mathrm{~K}\right)$ and $\mathrm{Co}_{2} \mathrm{FeSi}\left(T_{C}=1100 \mathrm{~K}\right)$ are rather promising [44]. However, it seems difficult to keep the high magnetoresistance in tunneling devices up to room temperature because of the instability of the compounds or alloys towards 
atomic diffusion and anti-site disorder at surfaces or tunnel junctions. This reduces the high spin polarization due to filling of the energy gap with states arising from the disorder. Regarding the MSMA, the difficulty is here that Curie temperatures and martensitic transformation temperatures are too low, preventing, for instance actuators to work at ambient temperatures.

Nevertheless, it may be interesting to evaluate $M(T)$ by using the calculated $J_{i j}$ in Monte Carlo simulations, which is shown in Fig. 8 for the case of $\mathrm{Ni}_{2} \mathrm{MnGa}$ (with the $J_{i j}$ taken for the $\mathrm{L} 2_{1}$ structure). Although, as discussed in Ref. [44], the calculated Curie temperatures of MSMA using mean-field theory agree to some extent with the experimental values. However, this is probably not a reliable procedure in case of MSMA with competing magnetic ordering tendencies. In this case, Monte Carlo simulations using the $J_{i j}$ obtained form zero-temperature $a b$ initio calculations will certainly yield better results, for details, see the discussion in Ref. [16]. Especially for the offstoichiometric systems like Ni-Mn-In, Ni-Mn-Sn and Ni-Mn-Sb, the AF interactions between the spin moments of Mn atoms on the Mn- and X-sublattice below $T_{M}$ not only compete with the FM ones but also change in a subtle way with temperature due to the temperature variation of the lattice constants. This, finally, is responsible for the complex magnetic phase diagram of In, Sn and Sb based off-stoichiometric Heusler systems, of which the Sb-system has been discussed in Ref. [41]. (note that a different picture of $\mathrm{AF}$ order in stoichiometric $\mathrm{Ni}_{2} \mathrm{MnGa}$ and $\mathrm{Ni}_{2} \mathrm{MnAl}$ was discussed by Enkovaara et al., where the AF order of the Mn spin moments leads to vanishing magnetization at the Ni sites [45]) Recent measurements of the $q$-dependence of the neutron scattering cross sections in the austenite $(500 \mathrm{~K})$ and martensite $(320 \mathrm{~K})$ states of $\mathrm{Ni}_{50} \mathrm{Mn}_{40} \mathrm{Sb}_{10}$ confirm this scenario and show that the FM reflections start to decrease in the martensitic phase and AF reflections start to increase [46].
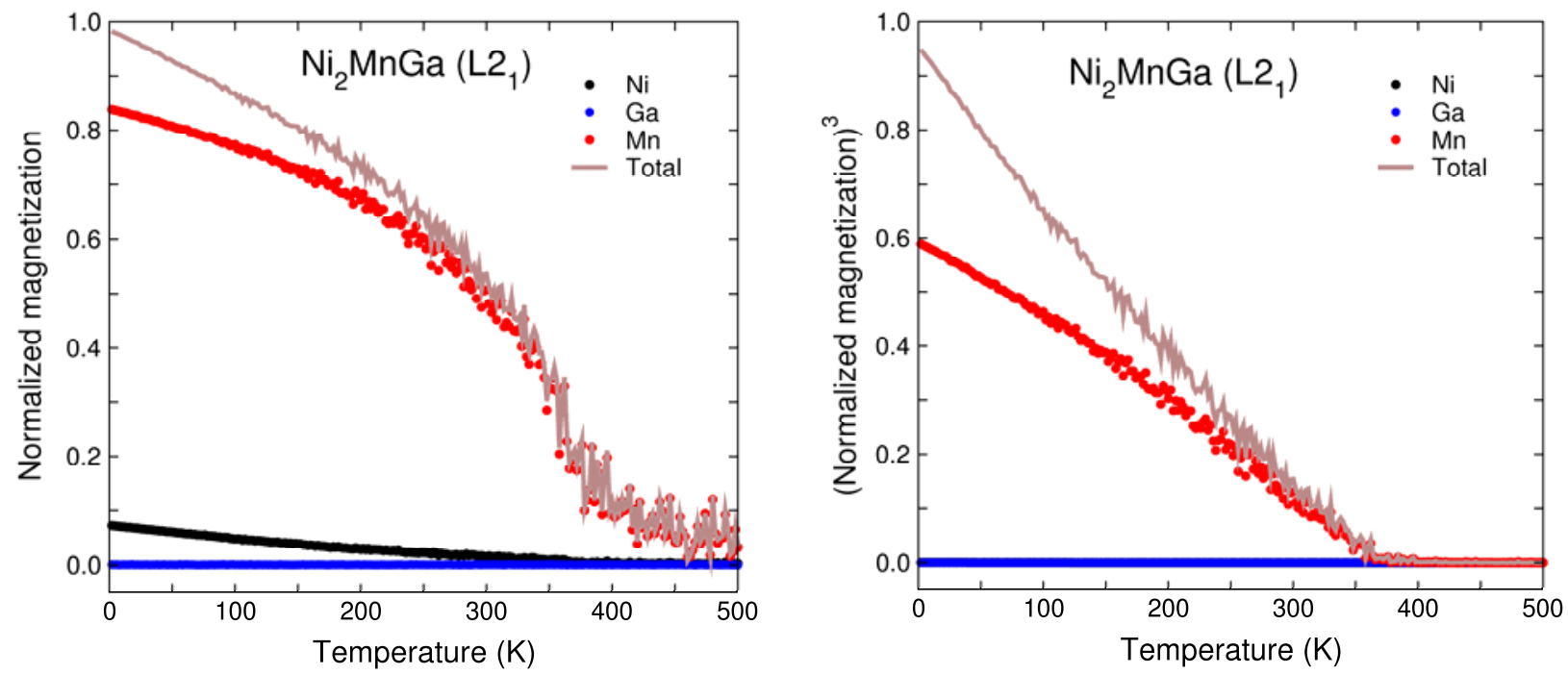

Fig. 8: Results of Monte Carlo simulations of the magnetization (normalized to $M /$ f.u.) using the zero-temperature $a b$ initio values of exchange coupling constants of cubic L2 ${ }_{1} \mathrm{Ni}_{2} \mathrm{MnGa}$ shown in Fig. 7. From the extrapolation of $M^{3}(T)$ to $M=0$, a Curie temperature of $375 \mathrm{~K}$ is estimated, which is in perfect agreement with the experimental value.

\section{Future of Spintronics, Magnetic Shape Memory and Magnetocaloric Systems}

As already suggested in this paper, combination of well-established spintronics materials with high Curie temperatures like $\mathrm{Co}_{2} \mathrm{MnGe}$ with MSMA and MCE materials like Ni-Mn-Z ( $\mathrm{Z}=\mathrm{Al}, \mathrm{Ga}$, In, $\mathrm{Sn}, \mathrm{Sb})$ may yield new and interesting phenomena like a near half-metal system undergoing a martensitic transformation, although we find the trend that half-metallicity and structural instability seem to exclude each other. Preliminary results for the DOS of NiCoMn(Ga, Ge) are shown in Fig. 4. The DOS in Fig. 4 seems to indicate that one would need a compound with $7.25<$ e/a $<7.5$ in 
order to observe a trend for both kinds of phenomena in one and the same system. Other details become important for the search of new materials like the influence of lattice vibrations, magnetic excitations, twin boundary motion under the impact of an external magnetic field etc. A few more details may be found in a recent review article where the influence of reduced magnetization at finite temperatures and the change of phonon spectra with external magnetic field is discussed on the basis of fixed spin moment calculations [47].

\section{References}

[1] P.J. Webster, K.R.A. Ziebeck, S.L. Town, and M.S. Peak, Phil. Mag. B 49, 295 (1984).

[2] P.J. Brown, A.Y. Bargawi, J. Crangle, K.-U. Neumann, and K.R.A. Ziebeck, J. Phys.: Condens. Matter 11, 4715 (1999).

[3] K. Ullakko, J.K. Huang, C. Kantner, R.C. O'Handley, and V.V. Kokorin, Appl. Phys. Lett. 69, 1966 (1996).

[4] A. Sozinov, A.A. Likhachev, N. Lanska, and K. Ullakko, Appl. Phys. Lett. 80, 1746 (2002).

[5] A. Sozinov, A.A. Likhachev, K. Ullakko, and V.K. Lindroos, J. Phys. (France) IV 112, 955 (2003).

[6] P. Müllner, V.A. Chernenko, and G. Kostorz, J. Appl. Phys. 95, 15331 (2004).

[7] A.A. Cherechukin, I.E. Dikshtein, D.I. Ermakov, A.V. Glebov, V.V. Koledov, D.A. Kosolapov, V.G. Sharov, A.A. Tulaikova, E.P. Krasnoperov, and T. Takagi, Phys. Lett. A 291, 175 (2001).

[8] Y. Sutou, N. Kamia, T. Omori, R. Kainuma, K. Ishida, and K. Oikawa, Appl. Phys. Lett. 84, 1275 (2004).

[9] F. Chen, X.L. Meng, W. Cai, L.C. Zhao, and G.H. Wu, J. Magn. Magn. Mater. 302, 459 (2006).

[10] P. Müllner, V.A. Chernenko, M. Wollgarten, and G. Kostorz, J. Appl. Phys. 92, 6708 (2002).

[11] P. Entel, V.D. Buchelnikov, M.E. Gruner, A. Hucht, V.V. Khovailo, S.K. Nayak, and A.T. Zayak, Mater. Sci. Forum 583, 21 (2008).

[12] I. Galanakis, P.H. Dederichs, and N. Papanikolaou, Phys. Rev. B 66, 174429 (2002).

[13] I. Galanakis, Ph. Mavropoulos, and P.H. Dederichs, J. Phys. D: Appl. Phys. 39, 765 (2006).

[14] S.A. Wolf, D.D. Awschalon, R.A. Buhrman, J.M. Daughton, S. von Molnar, M.L.Roukes, A.V. Chatelkanova, and D.M. Tragar, Science 294, 1488 (2001).

[15] I. Zutic, J. Fabian, and Das Sarma, Rev. Mod. Phys. 76, 323 (2004).

[16] V.D. Buchelnikov, P. Entel, S.V. Taskaev, V.V. Sokolovskiy, A. Hucht, M. Ogura, A. Akai, M.E. Gruner, and S.K. Nayak, Phys. Rev. B 78, 184427 (2008).

[17] S. Fujii, S. Ishida, and S. Asano, J. Phys. Soc. Jpn. 58, 3657 (1989).

[18] A. Ayuela, J. Enkovaara, K, Ullakko, and R.M. Nieminen, J. Phys.: Condens. Matter 11, 2017 (1999).

[19] A. Ayuela, J. Enkovaara, and R.M. Nieminen, J. Phys.: Condens. Matter 14, 5325 (2002).

[20] S.R. Barman, S. Banik, and A. Chakrabarti, Phys. Rev. B 72, 184410 (2005).

[21] P. Entel, V.D. Buchelnikov, V.V. Khovailo, A.T. Zayak, W.A. Adeagbo, M.E. Gruner, H.C. Herper, and E.F. Wassermann, J. Phys. D: Appl. Phys. 39, 865 (2006).

[22] S. Piozzi, A. Continenza, and A.J. Freeman, Phys. Rev. B 66, 094421 (2002). 
[23] I. Galanakis, and Ph. Mavropoulos, Phys. Rev. B 67, 104417 (2003).

[24] Half-Metallic Alloys: Fundamentals and Applications, edited by I. Galanakis and P. H. Dederichs, Lecture Notes in Physics, Vol. 676 (Springer, Berlin, 2005).

[25] R.A. de Groot, F.M. Mueller, P.G. van Engen, and K.H.J. Buschow, Phys. Rev. Lett. 50, 2024 (1983).

[26] G. Kresse and J. Furthmüller, Phys. Rev. B 54, 11169 (1996).

[27] G. Kresse and D. Joubert, Phys. Rev. B 59, 1758 (1996).

[28] P. Entel, E. Hoffmann, P. Mohn, K. Schwarz, and V.L. Moruzzi, Phys. Rev. B 47, 8706 (1993).

[29] D. Reinen and M. Atanasov, Magn. Reson. Rev. 15, 167 (1991).

[30] V. A. Chernenko, C. Seguí, E. Cesari, J. Pons, and V. V. Kokorin, Phys. Rev. B 57, 2659 (1998).

[31] A. Planes, Ll. Manosa, and M. Acet, J. Phys.: Condens. Matter 21, 233201 (2009).

[32] Yongbin Lee, Joo Yull Rhee, and B. N. Harmon, Phys. Rev. B 66, 054424 (2002).

[33] A. Zheludev, S.M. Shapiro, P. Wochner, and L.E. Tanner, Phys. Rev. B 54, 15045 (1996).

[34] U. Stuhr, P. Vorderwisch, V.V. Kokorin, and P.-A. Lindgard, Phys. Rev. B 56, 14360 (1997).

[35] Ll. Manosa, A. Planes, J. Zaretsky, T. Lograsso, D.L. Schlagel, and C. Stassis, Phys. Rev. B 64, 024305 (2001).

[36] S.M. Shapiro, P. Vorderwisch, K. Habicht, K. Hradil, and H. Schneider, Eur. Phys. Lett. 77, 56004 (2007).

[37] C. Bungaro, K.M. Rabe, and A. Dal Corso, Phys. Rev. B 68, 134104 (2003).

[38] A.T. Zayak, P. Entel, K.M. Rabe, W.A. Adeagbo, and M. Acet, Phys. Rev. B 72, 054113 (2005).

[39] A. T. Zayak, W. A. Adeagbo, P. Entel, and K. M. Rabe, Appl. Phys. Lett. 88, 111903 (2006).

[40] M.A. Uijttewaal, T. Hickel, J. Neugebauer, M.E. Gruner, and P. Entel, Phys. Rev. Lett. 102, 035702 (2009).

[41] M. Khan, I. Dubenko, S. Stadler, and N. Ali, J. Phys.: Condens. Matter, 20, 235204 (2008).

[42] Y. Kurtulus, R. Dronskowski, G.D. Samolyuk, and P. Antropov, Phys. Rev. B 71, 014425 (2005).

[43] E. Sasioglu, L.M. Sandratski, P. Bruno, and I. Galanakis, Phys. Rev. B 72, 184415 (2005).

[44] J. Thoene, S. Chadov, G. Fecher, C. Felser, and J. Kübler, J. Phys. D: Appl. Phys. 42, 084013 (2009).

[45] J. Enkovaara, A. Ayuela, J. Jalkanen, L. Nordström, and R.M. Nieminen, Phys. Rev. B 67, 054417 (2003).

[46] S. Aksoy, M. Acet, P.P. Deen, L. Manosa, and A. Planes, Phys. Rev. B 79, 212401 (2009).

[47] M.E. Gruner and P. Entel, J. Phys.: Condens. Matter. , accepted (2009). 